\title{
White matter hyperintensities on brain magnetic resonance in systemic sclerosis
}

\author{
F Sardanelli, A lozzelli, B Cotticelli, C Losacco, M Cutolo, A Sulli, F Nobili, G Rodriguez
}

Ann Rheum Dis 2005;64:777-779. doi: 10.1136/ard.2003.018283

\begin{abstract}
Objective: To evaluate the brain status of patients with systemic sclerosis (SSc).

Methods: Fourteen female patients with SSc aged 24-74, with a disease duration of 1-12 years and without other relevant systemic diseases, were enrolled. All patients and an age matched female control group (CG) of 14 clinically normal subjects, underwent brain magnetic resonance examination at $1.5 \mathrm{~T}$; spin echo proton density weighted images were evaluated. Mann-Whitney $U$ and Spearman rank correlation tests were used for statistical analysis.

Results: 170 white matter hyperintensities $\geqslant 2 \mathrm{~mm}$ in diameter were counted in the patient group (range 0-75, mean 12.1, median 4.5), only 13 in the $C G(0-2,0.9,1$, respectively), with a significant difference $(p=0.011)$. Moreover, 208 white matter hyperintensities $<2 \mathrm{~mm}$ were found in the patient group (0-38, 14.9, 8, respectively), only 31 in the CG $(0-7,2.0,1$, respectively), with a significant difference $(p=0.006)$. No statistically significant correlation between the number of hyperintensities and either patient's age or disease duration was observed.

Conclusion: White matter hyperintensities are more common in patients with SSc than in a CG. These findings might be related to obliterative microvascular processes due to the disease. Early brain involvement in patients with SSc may occur.
\end{abstract}

$\mathrm{S}$ ystemic sclerosis ( $\mathrm{SSc}$ ) is a progressive multisystem connective tissue disease of unknown aetiology, characterised by increased amounts of collagen in the blood vessels, skin, and visceral organs. Gastrointestinal tract, lungs, heart, and kidneys are mostly affected. The vascular changes begin with an injury to the endothelial cells and an intimal proliferation with fibrous thickening of the media, leading to obliterative microvascular disease. ${ }^{12}$

The prevalence and severity of vascular involvement of the central nervous system (CNS) in patients with SSc is still a matter of discussion. It is generally considered to be uncommon or as a secondary consequence of hypertension, uraemia, pulmonary dysfunction, and steroid treatment ${ }^{3}$; signs and symptoms of CNS involvement were infrequently reported in the large clinical series of patients with SSc of Tuffanelli and Winkelmann. ${ }^{4}$ Moreover, cerebrovascular lesions were not found more often in patients with SSc than in an age matched control group at pathological examination. ${ }^{5}$ However, several authors have reported CNS abnormalities in patients with SSc, dating from the pathological observations of Steven in $1898 .{ }^{6}{ }^{7}$ Cases of carotid and intracranial arteritis $^{8}$ have been reported, also. Neuropsychiatric manifestations, such as loss of memory, disorientation, depression, delusions, hallucinations, and reduced mental acuity were reported in the late phase of the disease, while transient ischaemic attacks, ischaemic stroke, and haemorrhage ${ }^{9}{ }^{10}$ have been described as a primary consequence of SSc in recent years.

Magnetic resonance (MR) imaging is considered to be the most sensitive diagnostic technique for detecting symptomatic and asymptomatic lesions in the brain, as demonstrated in multifocal diseases such as ischaemic encephalopathy and multiple sclerosis.

Thus, this study aimed at evaluating the brain status of patients with SSc by MR imaging.

\section{METHODS}

We studied 14 female patients with SSc (aged 24-74 years) with a disease duration of $1-12$ years, who had no serious diseases other than SSc. All the patients had received a diagnosis of SSc according to the criteria of the American Rheumatism Association. Exclusion criteria included severe or uncontrolled arterial hypertension; uncontrolled diabetes mellitus; relevant renal, respiratory, or hepatic failure; and severe anaemia. Three patients included were affected by mild arterial hypertension and were treated with angiotensin converting enzyme inhibitors or with a calcium blocking agent. One further patient was affected by moderate hypertension and was regularly treated with an angiotensin converting enzyme inhibitor and diuretics. Blood pressure was well controlled in these patients by the present treatment. None of the patients included was diabetic, but one showed impaired glucose tolerance. Renal function, as assessed by routine creatinine and blood urea nitrogen assays, was normal in all cases. These patients had no history of cerebrovascular accidents, head trauma, or other major neurological or psychiatric disease. Fourteen age matched clinically normal women were studied as a control group (CG).

Patients and normal subjects underwent brain MR examination at $1.5 \mathrm{~T}$ (Magnetom Vision, Siemens, Erlagen, Germany). After centring with a three step scout (axial, coronal, and mid-cerebral sagittal), a bicommissural paraaxial spin echo, double echo proton density and $\mathrm{T}_{2}$ weighted sequence was acquired with the following technical parameters: time of repetition/time of echo $=2500 / 20$ and 2500/ $80 \mathrm{~ms}$; pixel $0.98 \times 0.98 \mathrm{~mm}$; matrix $256 \times 256$; field of view $25 \mathrm{~cm}$ (rectangular 5-6/8); $22 \times 2$ para-axial slices with thickness of $3 \mathrm{~mm}$ (no gap, two interleaved acquisitions), for a total of 44 proton density and $44 \mathrm{~T}_{2}$ weighted images. A spin echo $T_{1}$ weighted sequence was acquired for clinical purposes.

Only proton density images were used for counting focal brain abnormalities both in patients with SSc and the CG, owing to the higher sensitivity for periventricular lesions in white matter inflammatory diseases in comparison with $T_{2}$ weighted images. We are aware that fluid attenuated inversion recovery is more sensitive than spin echo proton

Abbreviations: CG, control group; CNS, central nervous system; MR, magnetic resonance; $\mathrm{SSc}$, systemic sclerosis 


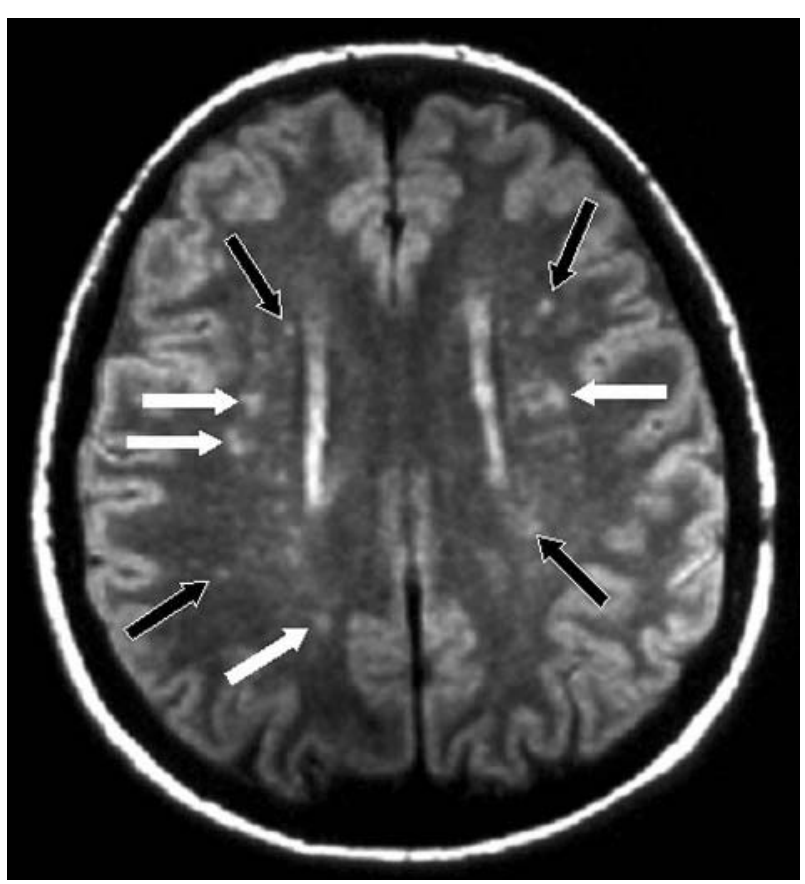

Figure 1 Proton density weighted brain MR image with major hyperintensities ( $\geqslant 2 \mathrm{~mm}$ in diameter; white arrows) and minor hyperintensities ( $<2 \mathrm{~mm}$ in diameter; black arrows) in the deep white matter of a patient with SSc.

density and $\mathrm{T}_{2}$ weighted sequences in detecting supratentorial periventricular inflammatory lesions; however, taking into account the low sensitivity of fluid attenuated inversion recovery in detecting subtentorial brain stem inflammatory lesions, proton density weighted sequences were finally preferred and used in this study.

Two readers counted the white matter hyperintensities in a randomised order, without knowing the patient/control status of each subject, using a remote console (Magic View, Siemens, Erlangen, Germany). All the abnormalities were electronically measured along the longest diameter to distinguish those $\geqslant 2 \mathrm{~mm}$ from those $<2 \mathrm{~mm}$ in diameter.

For statistical analysis, the Mann-Whitney $U$ test and Spearman rank correlation coefficient were used.

\section{RESULTS}

One hundred and seventy white matter major hyperintensities ( $\geqslant 2 \mathrm{~mm}$ in diameter) were counted in the patient group (range $0-75$, mean 12.1 , median 4.5 ), only 13 in the CG (range 0-2, mean 0.9, median 1), with a significant difference $(p=0.011$, Mann-Whitney) between the two
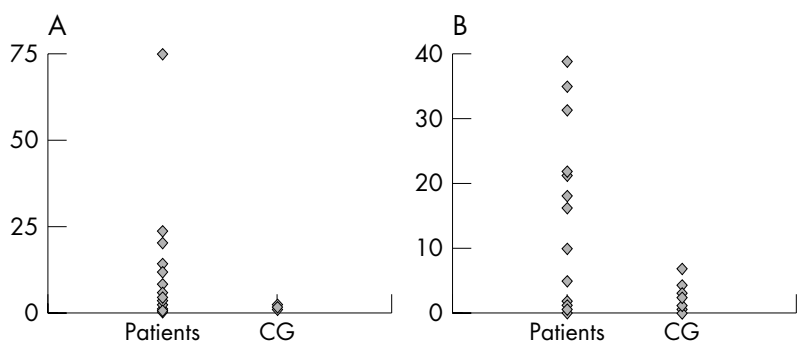

Figure 2 Distribution of the number of $(A)$ major ( $\geqslant 2 \mathrm{~mm}$ in diameter) and (B) minor hyperintensities ( $<2 \mathrm{~mm}$ in diameter) on brain $M R$ imaging in 14 patients with SSc and in an age matched control group (CG). A significant difference was found for both major $(p=0.011)$ and minor hyperintensities $(p=0.006)$. groups. Among the major white matter hyperintensities, 64 hyperintensities $\geqslant 5 \mathrm{~mm}$ in diameter were found in eight patients ( 28 hyperintensities in seven patients and 36 in one patient) and none in the CG. Moreover, 208 white matter minor hyperintensities $(<2 \mathrm{~mm}$ in diameter) were found in the patient group $(0-38,14.9,8$, respectively), only 31 in the CG $(0-7,2.0,1$, respectively), with a significant difference $(p=0.006$, Mann-Whitney). Figure 1 shows examples of minor and major hyperintensities. Figure 2 shows graphically the distribution of the number of major and minor hyperintensities in patients and the CG. An outlier with 77 major and 38 minor hyperintensities is clearly visible. Calculation of the $p$ values excluding the outlier was performed, still showing significant differences between patients and CG $(p=0.019$ for major and $p=0.029$ for minor hyperintensities).

No statistically significant correlation between the number of hyperintensities and both patient's age and disease duration was seen.

\section{DISCUSSION}

Our study demonstrated that white matter hyperintensities are more common in patients with SSc than in the CG. The number and size of the hyperintensities suggest abnormalities in the territories supplied by the smaller branches and the perforating vessels. These findings confirm the hypothesis of a previous direct microangiopathic injury of the brain $^{11}{ }^{12}$ and are in accordance with previously described brain involvement in four cases of a particular cutaneous form of SSc, the so-called "en coup de sabre" linear scleroderma $^{13-15}$ (in all four cases MR imaging demonstrated brain hyperintensities), even though Stone et al performed brain biopsy and found "evidence of an inflammatory process which may be amenable to immunosuppressive treatment". ${ }^{15}$

We know that the underlying pathogenesis of these brain abnormalities could be explained by a range of different mechanisms, which might act individually, or together, in producing the lesions. The first of these proposed pathways are non-immune reactions, with cell mediated activity and autoantibody production likely to account for the isolated cases of vasospasm (and associated autonomic dysfunction). The second mechanism is vascular, with changes in endothelial cells and basement membrane, causing a noninflammatory microangiopathy of the vasa nervorum, a tendency for platelets to aggregate, and a final ischaemic CNS injury-although the initial precise causal event remains unclear. The third pathway consists of alterations of the collagen metabolism, proliferation of specific fibroblast subpopulations, and invasion into tissue and fibrosis, though this seems unlikely to produce CNS abnormalities because of the paucity of connective tissue in the brain. The role of cerebral vasculitis in SSc remains unknown, as previous necropsy reports have rarely described such alterations. ${ }^{8}$ All these reports and hypotheses deserve further investigation, both histologically and clinically, to elucidate better the pathophysiology of CNS involvement in SSc.

In conclusion, our study shows that brain MR hyperintensities are more common in patients with SSc than in the CG, supporting the hypothesis of an early and frequent brain involvement in patients with SSc.

\section{Authors' affiliations}

F Sardanelli, A lozzelli, B Cotticelli, Department of Diagnostic Imaging, Istituto Policlinico San Donato, San Donato Milanese, Milan, Italy C Losacco, Department of Radiology, San Martino Hospital, Genoa, Italy M Cutolo, A Sulli, Department of Internal Medicine, Division of Rheumatology, University of Genoa, Italy

F Nobili, G Rodriguez, Clinical Neurophysiology, Department of Endocrinological and Metabolic Sciences, University of Genoa, Italy 
Correspondence to: Professor F Sardanelli, Department of Diagnostic Imaging, University Hospital Istituto Policlinico San Donato, Via Morandi 30, 20097 San Donato Milanese, Milan, Italy; f.sardanelli@ grupposandonato.it

Accepted 10 September 2004

\section{REFERENCES}

1 Pearson JD. The endothelium: its role in scleroderma. Ann Rheum Dis 1991;50:866-71.

2 Blann AD, Illingworth K, Layson MIV. Mechanisms of endothelial damage in systemic sclerosis and Raynaud's phenomenon. J Rheumatol 1993;20:1325-30.

3 Seibold JR. Scleroderma. In: Kelley WN, Harris ED Jr, Ruddy S, Sledge CB, eds. Textbook of rheumatology. 5th ed. Philadelphia: Saunders, 1997: 1133-62

4 Tuffanelli D, Winkelmann RK. Systemic scleroderma: clinical study of 727 cases. Arch Dermatol 1961;84:359-71.

5 D'Angelo WA, Fries JF, Masi AT, Shulman LE. Pathologic observations in systemic sclerosis (scleroderma). Am J Med 1969;46:428-40.

6 Steven JL. Case of scleroderma with pronounced hemiatrophy of the face, body and extremities -death from ovarian tumor-account on the postmortem examination: a sequel. Glasgow Med J 1898;50:401-22.
7 Piper WN, Helwig EB. Progressive systemic sclerosis. Arch Dermatol 1955;72:535-46.

8 Pathak R. Gabor AJ. Scleroderma and central nervous system vasculitis. Stroke 1991;22:410-13.

9 Andonopoulos AP, Maraziotis T, Yarmenitis S, Papapetropoulos T. Multiple spontaneous intracerebral hemorrhages in a patient with progressive systemic sclerosis. Rev Rheum (Engl Ed) 1998;65:437-40.

10 Yukawa H, Kubo Y, Otawara Y, Kudo A, Tomitsuka N, Yoshida K, et al. A case of left occipital lobe hemorrhage in a patient with progressive systemic sclerosis: evaluation of cerebral angiography and histology. No Shinkei Geka 2000;28:1003-7.

11 Cutolo M, Nobili F, Sulli A, Pizzorni C, Briata M, Faelli F, et al. Evidence of cerebral hypoperfusion in scleroderma patients. Rheumatology (Oxford) 2000;39:1366-73.

12 Cutolo M, Sulli A, Pizzorni C, Accardo S. Nailfold videocapillaroscopy assessment of microvascular damage in systemic sclerosis. J Rheumatol 2000;27:155-60

13 Blaszczyk M, Krolicki L, Krasu M, Glinska O, Jablonska S. Progressive facial hemiatrophy: central nervous system involvement and relationship with scleroderma en coup de sabre. J Rheumatol 2003;30:1997-2004.

14 Chung MH, Sum J, Morrell MJ, Horoupian DS. Intracerebral involvement in scleroderma en coup de sabre: report of a case with neuropathologic findings. Ann Neurol 1995; 37:679-81.

15 Stone J, Franks AJ, Guthrie JA, Johnson MH. Scleroderma "en coup de sabre": pathological evidence of intracerebral inflammation. J Neurol Neurosurg Psychiatry 2001;70:382-5. 